\title{
Effect of TIMP1 transfection on PTEN expression in human kidney proximal tubular cells
}

\author{
J.X. Chen', G.Y. Cai ${ }^{2}$, X.M. Chen², H. Liu' ${ }^{1}$ X. Chen', Y.M. Peng ${ }^{2}$, F.Y. Liu', Z. Li \\ and S.Z. Shi ${ }^{1}$ \\ 1'Department of Nephrology, the Second Xiangya Hospital of Central South University, \\ Changsha, China \\ ${ }^{2}$ Department of Geriatric Nephrology, General Hospital of PLA, Beijing, China \\ Corresponding author: F.Y. Liu \\ E-mail: chenjunxianghn@163.com \\ Genet. Mol. Res. 14 (4): 17373-17383 (2015) \\ Received August 13, 2015 \\ Accepted October 6, 2015 \\ Published December 21, 2015 \\ DOI http://dx.doi.org/10.4238/2015.December.21.6
}

ABSTRACT. To explore the role of metalloproteinase-1 (TIMP-1) tissue inhibitor in the mechanisms of kidney aging, we observed the effects of sense and antisense transfection of TIMP-1 and of metalloproteinase (MMP) inhibitors on phosphatase and tensin homolog (PTEN), vascular endothelial growth factor (VEGF), and Flk-1 expression in TIMP-1 transgenic human proximal tubular epithelial cells (HKCs). Transfected HKCs were co-incubated with $100 \mu \mathrm{M}$ MMP-2 and MMP-9 inhibitor III for 24 $\mathrm{h}$ to affect enzyme inhibition. TIMP-1, MMP-2, MMP-9, PTEN, VEGF, and Flk-1 mRNA expression was detected by reverse transcription-polymerase chain reaction. PTEN, VEGF, and Flk-1 protein expression in cells of each experimental group was measured by indirect immunofluorescence. We found that PTEN expression was up-regulated $(P<0.05)$ in the sense TIMP-1-transfected group $(P<0.05)$ compared with the non-transfected and empty vector groups, and that expression of VEGF and Flk-1 was down-regulated $(P<0.05)$. In contrast, the antisense TIMP-1 transgenic group showed the opposite results $(P<0.05)$. No significant differences 
in expression of PTEN, VEGF, or Flk-1 were observed among the MMP2/MMP-9 inhibitor III, non-transfected, and empty vector groups $(P>$ $0.05)$. These results suggest that in the progression of renal aging, high expression of TIMP-1 up-regulates PTEN expression through an MMPindependent pathway, and subsequently down-regulates the expression of VEGF and Flk-1, indicating that PTEN and TIMP-1 are involved in the aging-associated impairment of renal angiogenesis. Our study provides a theoretical basis for further exploration of the mechanism underlying TIMP1 participation in renal aging progression.

Key words: TIMP-1; Kidney aging; Angiogenesis; PTEN

\section{INTRODUCTION}

Vascular endothelial growth factor (VEGF) is a crucial pro-angiogenic factor that plays an important role in angiogenesis. It mainly acts by interacting with the receptor Flk-1, further initiating intracellular signal transduction, and thereby promoting endothelial cell proliferation, migration, and angiogenesis (Kang et al., 2002; Huang et al., 2012). Research has revealed that highly expressed sense and antisense TIMP-1 might be involved in age-related impairment of angiogenesis via up-regulating phosphatase and tensin homolog (PTEN) in the aging kidney. However, whether highly expressed sense and antisense TIMP-1 can also exert an impact on disordered age-related angiogenesis by down-regulating VEGF/FIk-1 has not yet been elucidated. In this study, sense and antisense TIMP-1 recombinant expression vectors were transfected into human kidney proximal tubular cells (HKCs) followed by simultaneous treatment of matrix metalloproteinase (MMP)-2/MMP-9-specific enzyme inhibitors. The impact of sense and antisense TIMP-1 transfection and enzyme inhibitor interference on HKCs were observed to explore the underlying mechanisms of TIMP-1 on the aging kidney.

\section{MATERIAL AND METHODS}

\section{Reagents}

MMP-2/MMP-9 inhibitor III was obtained from Merck Pharmaceuticals (Kenilworth, NJ, USA); goat MMP-9/MMP-2 polyclonal antibody, rabbit TIMP-1 polyclonal antibody, rabbit VEGF polyclonal antibody, rabbit $\beta$-actin polyclonal antibody, mouse anti-human PTEN monoclonal antibody, FITC-labeled goat anti-mouse IgG, and goat anti-rabbit IgG were from Santa Cruz Biotechnology (Dallas, TX, USA ); horseradish peroxidase-labeled goat anti-rabbit IgG, rabbit anti-goat IgG, and goat rabbit anti-mouse IgG were also from Santa Cruz Biotechnology; M-MLV reverse transcriptase was from Invitrogen Life Technologies (Carlsbad, CA, USA); human and mouse TIMP-1, mouse MMP2, mouse MMP9, human PTEN, and VEGF primers were synthesized by SBS Genetech Co., Ltd. (Beijing, China).

\section{Cell culture and establishment of a cell line with stable expression of TIMP-1}

HKCs were stored in the Department of Nephrology, Chinese PLA General Hospital. Three HKC cell lines stably transfected with pcDNA 3.0, TIMP-1S-pcDNA 3.0, and TIMP-1AS-pcDNA 3.0 (pcDNA 3.0 recombinant plasmid containing full-length sense and antisense human TIMP- 
$1 \mathrm{cDNA}$ ) were previously constructed in the Molecular Biology Research Center of the Second Xiangya Hospital of Central South University as described (Lin Hongli et al., 2001). The three established cell lines were referred to as 1) normal non-transfected cells (parental/HKCs); 2) empty vector transfected cells (vector/HKCs); 3 ) sense TIMP-1 transfected cells (TIMP-1S/HKCs); and 4) antisense TIMP-1 transfected cells (TIMP-1AS/HKCs). The HKCs were cultured and passaged in DMEM medium containing $10 \%$ fetal bovine serum (FBS), $100 \mathrm{U} / \mathrm{mL}$ penicillin, and $100 \mathrm{U} / \mathrm{mL}$ streptomycin at $37^{\circ} \mathrm{C}$, with $5 \% \mathrm{CO}_{2}$ in a humid atmosphere.

\section{Construction and identification of plasmids}

The PTEN siRNA plasmid and control siRNA plasmid were donated by Professor Huang Boqu of Northeastern University of China. The RNAi target sequences of human PTEN (5' - GAC TTG AAG GCG TAT ACA G - 3') were designed according to the full-length human PTEN gene according to the GenBank database. The oligonucleotide sequences that encoded the chosen siRNA sequence were designed and inserted into the pSilencer 2.0-U6 vector. The plasmid (pSilencer 2.0U6 Vector) was sequenced and confirmed by Santa Cruz Biotechnology, China (Figure 1).

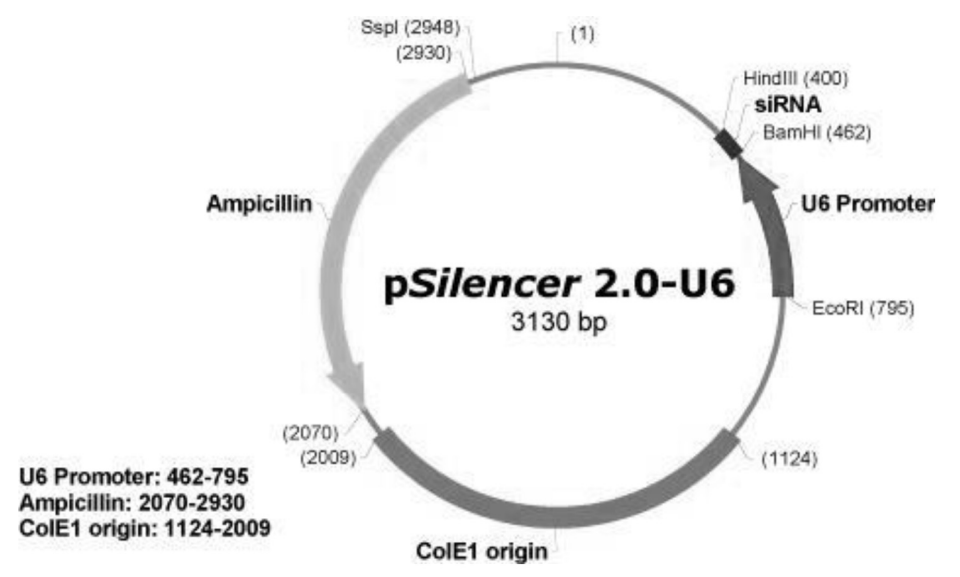

Figure 1. pSilencer 2.0-U6 vector map.

\section{Experimental design, grouping, and liposome-mediated transfection of plasmid DNA}

\section{Experimental groups}

In order to observed the impact of the sense and antisense TIMP-1 transfection and enzyme inhibitor interference on the expression of PTEN and VEGF in HKCs, the cells were divided into five groups: 1. normal non-transfected group (parental); 2. empty vector of the transfected group (vector); 3. sense TIMP-1 transfected group (TIMP-1S); 4. antisense TIMP-1 transfected group (TIMP-1AS); 5. normal non-transfected MMP inhibitor group (inhibitor III $100 \mu \mathrm{m}$ ) (Akahane et al., 2004).

Cells in each group were seeded in cell culture flasks (surface area $25 \mathrm{~cm}^{2} ; 5$ flasks per sample) at a density of $5 \times 10^{4} / \mathrm{mL}$, and were cultured in DMEM medium containing $10 \% \mathrm{FBS}$ and $100 \mathrm{U} / \mathrm{mL}$ penicillin and streptomycin in a humid atmosphere of $95 \%$ air and $5 \% \mathrm{CO}_{2}$ at $37^{\circ} \mathrm{C}$. Upon reaching 60 to $80 \%$ confluence, the cells were washed with phosphate-buffered saline and sub-cultured with serum-free DMEM for $16 \mathrm{~h}$. Subsequently, the cells were incubated with DMEM 
plus $10 \%$ FBS or RPMI1640 medium with or without $100 \mu$ M MMP-2/MMP-9 inhibitor III for $24 \mathrm{~h}$. The cells and the culture supernatant was harvested and analyzed for the presence of cytokines.

The PTEN siRNA was transfected into HKCs stably expressing human TIMP-1S for further observation. There were three experimental groups: A. sense TIMP-1 transfected group (TIMP1S); B. PTEN siRNA group (PTEN siRNA plasmid transfected HKCs stably expressing human TIMP-1S); and C. control siRNA group (control siRNA plasmid transfected HKCs stably expressing human TIMP-1S). Three replicate wells were used for each group.

\section{Liposome-mediated transfection of plasmid DNA}

Twenty-four hours prior to transfection, cells in the TIMP-1S group in logarithmic growth phase were seeded into 6-well plates and cultured in DMEM containing 10\% FBS, or RPMI1640 medium consisting of $100 \mathrm{U} / \mathrm{mL}$ penicillin and streptomycin in a humid atmosphere of 95\% air and $5 \% \mathrm{CO}_{2}$ at $37^{\circ} \mathrm{C}$ until the cells were grown to $40-60 \%$ confluence. The culture medium was replaced $1 \mathrm{~h}$ before transfection with $2 \mathrm{~mL}$ fresh medium per well and culturing was continued. For transfection, $5 \mu \mathrm{g}$ PTEN siRNA or control siRNA plasmid was added into normal saline (total volume $100 \mu \mathrm{L}$ ), mixed thoroughly, and incubated for $5 \mathrm{~min}$ at room temperature. Simultaneously, 2 $\mu \mathrm{g}$ Vigofect was added into normal saline (total volume $100 \mu \mathrm{L}$ ) followed by thorough mixing and 5 min incubation at room temperature. Then the diluted Vigofect mixture was added drop by drop into the diluted DNA solution accompanied with gentle vortexing. After 15 min incubation, the reaction mixture was evenly added into $2 \mathrm{~mL}$ cell culture medium and incubated for $6 \mathrm{~h}$. The medium was replaced with fresh medium and incubated for another $48 \mathrm{~h}$ before harvesting.

\section{PTEN and VEGF expression detection by indirect immunofluorescence}

The cells in the five study groups: parental, vector, TIMP-1S, TIMP-1AS, and inhibitor III $100 \mu \mathrm{M}$ groups, were treated according to kit instructions and were observed under a confocal laser scanning microscope. In each sample, 20 cells were randomly selected and the mean fluorescence intensities of PTEN and VEGF positive cells were measured using the Image-Pro Plus 4.5 software (Media Cybernetics, Rockville, MD, USA), and the mean value of the expression levels of PTEN and VEGF were used to evaluate their overall expression.

\section{TIMP-1, MMP-2, MMP-9, PTEN, and VEGF mRNA expression evaluated using reverse transcription-polymerase chain reaction (RT-PCR)}

Total RNA was extracted using the TRIzol reagent. RT-PCR primer sequences are shown in Table 1. GAPDH was used as the internal control. Agarose gel electrophoresis was used to detect the PCR product: $8 \mu \mathrm{L}$ each PCR product was electrophoresed on a $1.5 \%$ agarose gel and the images were captured and semi-quantitatively analyzed using an imaging system (Alphalmager, 2200, Alpha Innotech, Santa Clara, CA, USA).

\section{Statistical analyses}

Statistical analysis was performed using the SPSS 15.0 software (SPSS Inc., Chicago, IL, USA). Data are reported as means \pm standard deviation. Univariate analysis of variance was used to compare mean differences in the study groups. $P$ values less than 0.05 were considered to be statistically significant. 
Table 1. Primer sequences used for reverse transcription-polymerase chain reaction.

\begin{tabular}{|c|c|c|c|}
\hline Protein & Primer sequence & Fragment length (bp) & Annealing temperature $\left({ }^{\circ} \mathrm{C}\right)$ \\
\hline TIMP-1 & $\begin{array}{l}\text { Upstream: 5' - CAG ACC ACC TTA TAC CAG CGT - 3' } \\
\text { Downstream: 5' - GAT AAA CAG GGA AAC ACT GTG C - 3' }\end{array}$ & 311 & $60.0^{\circ}$ \\
\hline MMP-2 & $\begin{array}{l}\text { Upstream: 5' - AGA CCC CTG GCT TTT CAC TGC TGG - 3' } \\
\text { Downstream: } 5^{\prime} \text { - GTT TCC GCT TCT GGC TGG GTC TGT - 3' }\end{array}$ & 412 & $59.0^{\circ}$ \\
\hline MMP-9 & $\begin{array}{l}\text { Upstream: 5' - CGC GCT GGG CTT AGA TCA TTC CTC - } 3^{\prime} \\
\text { Downstream: } 5^{\prime} \text { - GCA GCG CGG GCC ACT TGT C - 3' }\end{array}$ & 476 & $62.0^{\circ}$ \\
\hline PTEN & $\begin{array}{l}\text { Upstream: 5' - GAT GGC ACT TTC CCG TTT TA - 3' } \\
\text { Downstream: 5' - TCT GAG CAT TCC CTC CAT TC - 3' }\end{array}$ & 254 & $55.0^{\circ}$ \\
\hline VEGF & $\begin{array}{l}\text { Upstream: 5' - TCC GGA TGG GCA CGA GTA - 3' } \\
\text { Downstream: 5' - TGG CGG AGA GGG GCA CAT - 3' }\end{array}$ & 121 & $61.0^{\circ}$ \\
\hline Flk-1 & $\begin{array}{l}\text { Upstream: 5' - GGA ACG CTA CCC CGC ACG AAT GAT - 3' } \\
\text { Downstream: 5' - GGT CCT GGC CCG CAA AGA AGT C - 3' }\end{array}$ & 186 & $66.0^{\circ}$ \\
\hline GAPDH & $\begin{array}{l}\text { Upstream: 5' - TGC ACC ACC AAC TGC TTA GC - 3' } \\
\text { Downstream: 5' - GGC ATG GAC TGT GGT CAT GAG - 3' }\end{array}$ & 191 & $58.0^{\circ}$ \\
\hline
\end{tabular}

\section{RESULTS}

\section{Identification of PTEN siRNA plasmid DNA}

A gene fragment for insertion of $62 \mathrm{bp}$ was generated based on literature review and plasmid map creation. The sequencing result showed that the oligonucleotide inserted had the sequence of 5' - GAC TTG AAG GCG TAT ACA G - 3', which was consistent with the target DNA sequence (Figure 2).

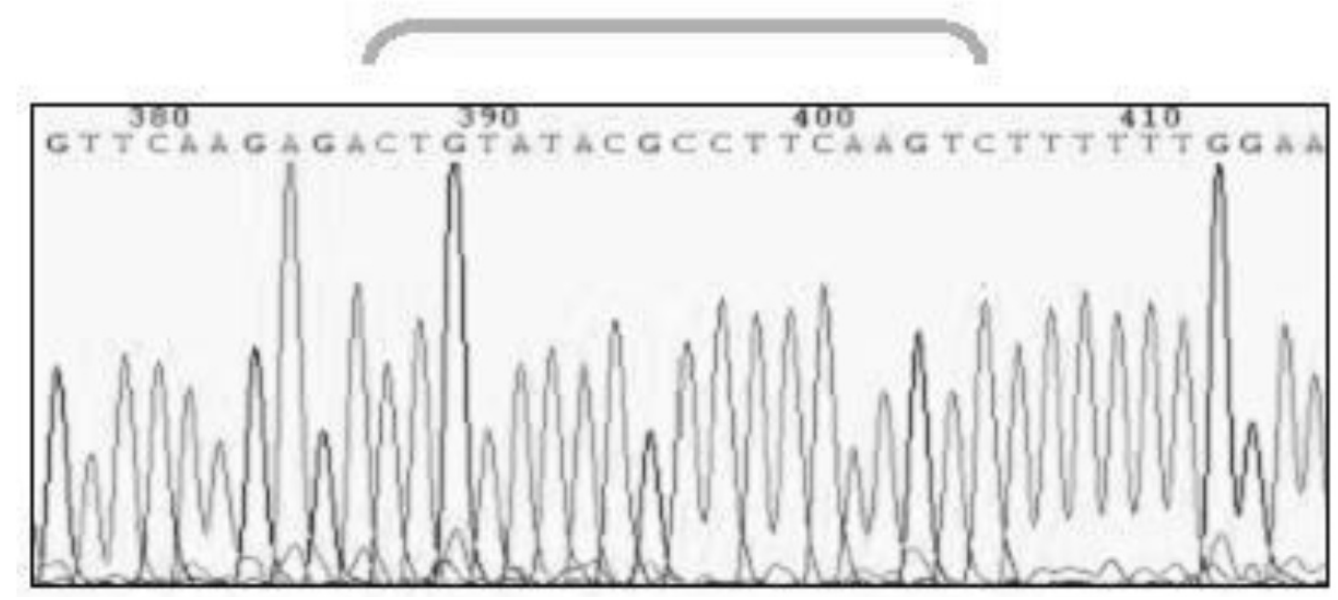

Figure 2. Target DNA sequence insert.

\section{Identification of exogenous DNA in HKCs}

Specific primers for exogenous gene identification were designed according to the vectors with exogenous DNA sequence. PCR results showed that the exogenous DNA was integrated into the genome of HKCs. In addition, a neo gene was successfully included in vector/HKC, TIMP-1S/HKC, and TIMP-1AS/HKC groups, and no inclusion was found in parental/HKCs. Inclusion of the antisense TIMP1 gene was observed in TIMP-1AS/HKCs, which was not identified in other cells (Figure 3). 

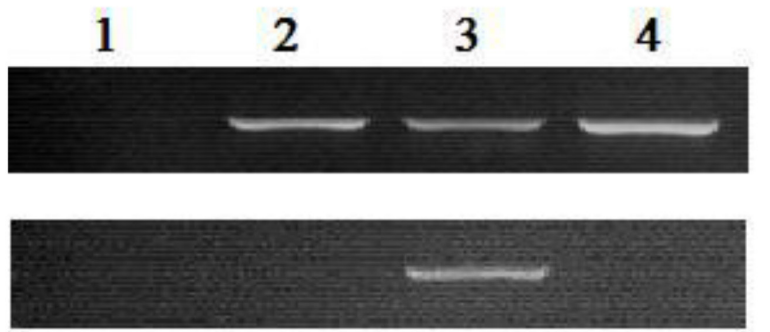

B

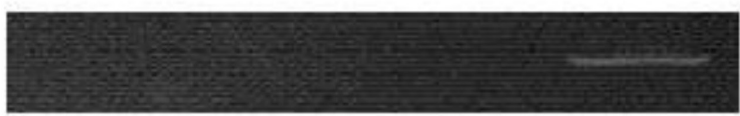

C

Figure 3. Identification of exogenous DNA in HKCs. Lanes 1-4 indicate parental/HKCs, vector/HKCs, TIMP-1S/HKCs, and TIMP-1AS/HKCs, respectively. Row A: stable transfected pcDNA3.0; B: TIMP-1S-pcDNA3.0; and C: TIMP-1ASpcDNA3.0. HKCs, human kidney cells.

\section{Detection of PTEN, VEGF, and Flk-1 expression with indirect immunofluorescence}

\section{Effect on PTEN expression in HKCs}

The results showed the strongest expression of PTEN in the TIMP-1S/HKCs $(P<0.05)$, and the weakest expression in the TIMP-1AS/HKCs $(P<0.05)$. Nevertheless, no significant difference in PTEN expression was found between parental/HKCs, vector/HKCs, and MMP-2/ MMP-9 inhibitor III $(100 \mu M)$ HKCs $(P>0.05)$. Detailed information is shown in Figure 4.
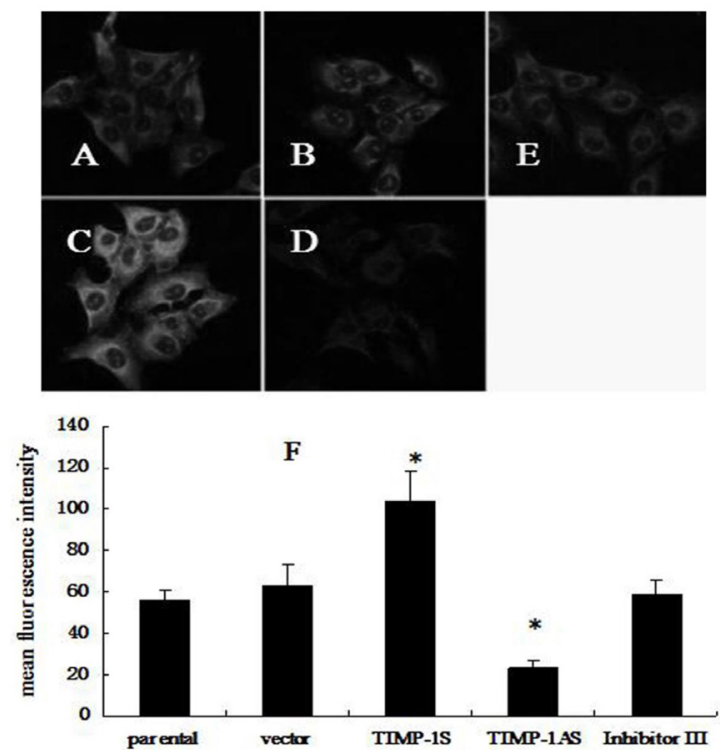

Figure 4. PTEN expression as measured by indirect immunofluorescence in each group. A. Parental/HKCs; B. vector/ HKCs; C. TIMP-1S/HKCs; D. TIMP-1AS/HKCs; E. MMP-2/MMP-9 inhibitor III (100 $\mu$ M); and F. histogram of the mean fluorescence intensity of PTEN in each group. ${ }^{*} \mathrm{P}<0.05$ compared with groups of parental/HKCs, vector/HKCs, and inhibitor III/HKCs $(100 \mu \mathrm{M}) ; 600 X$. HKCs, human kidney cells. 


\section{Effect on VEGF expression in HKCs}

The results showed that the weakest signal of VEGF expression was detected in the TIMP$1 \mathrm{~S} / \mathrm{HKCs}(\mathrm{P}<0.05)$, and the strongest in the TIMP-1AS/HKCs $(P<0.05)$. However, no significant difference in VEGF expression was observed between parental/HKCs, vector/HKCs, and MMP-2/ MMP-9 inhibitor III $(100 \mu \mathrm{M})$ HKCs $(\mathrm{P}>0.05)$. The results are shown in Figure 5.
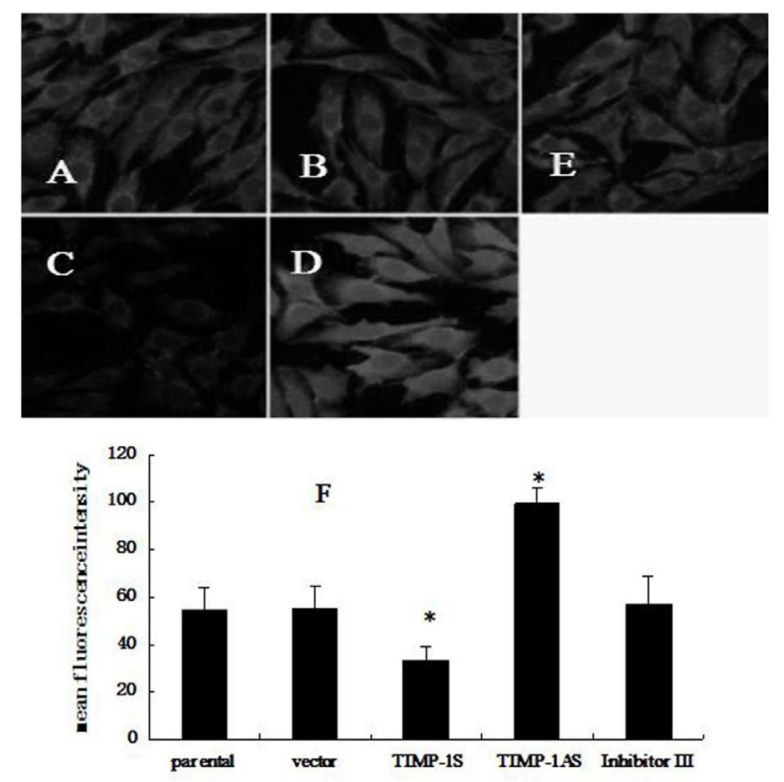

Figure 5. VEGF expression as measured by indirect immunofluorescence in each group. A. Parental/HKCs; B. vector/ HKCs; C. TIMP-1S/HKCs; D. TIMP-1AS/HKCs; E. MMP-2/MMP-9 inhibitor III (100 $\mu \mathrm{M})$; and F. histogram of the mean fluorescence intensity of PTEN in each group. ${ }^{*} \mathrm{P}<0.05$ compared with groups of parental/HKCs, vector/HKCs, and inhibitor III/HKCs (100 $\mu \mathrm{M})$; 600X. HKCs, human kidney cells.

\section{RT-PCR test results}

\section{Expression of TIMP-1, MMP-2, and MMP-9 mRNA in HKCs}

The study revealed higher expression of TIMP-1, MMP-2, and MMP-9 mRNA in TIMP-1S/ HKCs and TIMP-1AS/HKCs than in parental/HKCs, vector/HKCs, and MMP-2/MMP-9 inhibitor III $(100 \mu \mathrm{M}) \mathrm{HKCs}(\mathrm{P}<0.05)$. Nevertheless, the difference in expression of TIMP-1, MMP-2, and MMP9 mRNA was not significantly different between parental/HKCs, vector/HKCs, and MMP-2/MMP-9 inhibitor III $(100 \mu \mathrm{M})$ HKCs $(\mathrm{P}>0.05)$, which is displayed in Figure 6.

\section{VEGF mRNA expression in HKCs}

Among the five groups, TIMP-1S/HKCs presented a minimal expression of VEGF mRNA $(P<0.05)$, and TIMP-1AS/HKCs showed the maximum value $(P<0.05)$. Nevertheless, there was no significant difference in expression of VEGF mRNA between parental/HKCs, vector/HKCs, and MMP-2/MMP-9 inhibitor III $(100 \mu \mathrm{M}) \mathrm{HKCs}(\mathrm{P}>0.05)$. The findings are shown in Figure 7. 


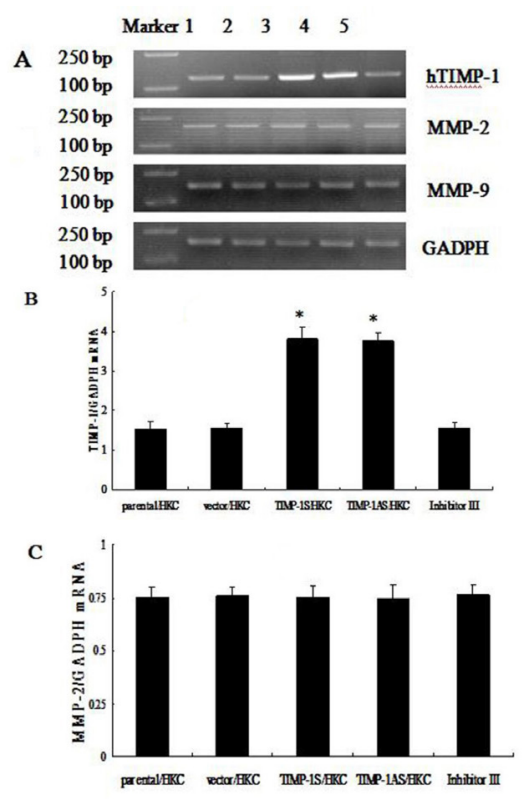

D

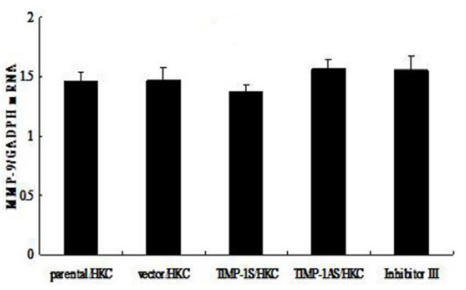

Figure 6. Expression of TIMP-1, MMP-2, and MMP-9 mRNA. A. Lane 1: parental/HKCs, lane 2: vector/HKCs, lane 3: TIMP-1S/HKCs, lane 4: TIMP-1AS/HKCs, and lane 5: MMP-2/MMP-9 inhibitor III (100 $\mu \mathrm{M})$. B.-D. Histograms for image analysis results; ${ }^{*} \mathrm{P}<0.05$, compared with parental/HKCs, vector/HKCs, and inhibitor III/HKCs (100 $\left.\mu \mathrm{M}\right)$. HKCs, human kidney cells.

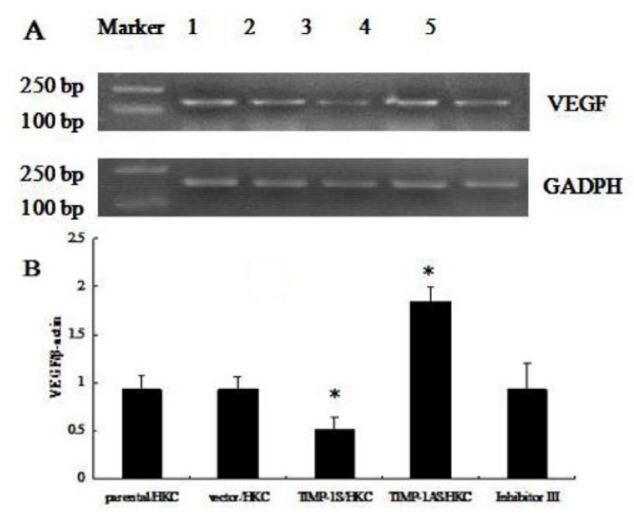

Figure 7. VEGF mRNA expression in HKCs. A. Lane 1: parental/HKCs, lane 2: vector/HKCs, lane 3: TIMP-1S/HKCs, lane 4: TIMP-1AS/HKCs, lane 5: MMP-2/MMP-9 inhibitor III $(100 \mu \mathrm{M})$. B. Histogram of image analysis results. *P < 0.05, compared with parental/ HKCs, vector/HKCs, and inhibitor III/HKC $(100 \mu \mathrm{M})$. HKCs, human kidney cells. 


\section{Expression of TIMP-1, PTEN, and VEGF mRNA in PTEN siRNA and control siRNA transfected TIMP-1S/HKCs}

The results showed significantly lower PTEN mRNA expression in the PTEN siRNA group than the TIMP-1S/HKCs and control siRNA group. The latter two groups, however, had no significant difference in PTEN mRNA expression ( $P>0.05)$. Furthermore, VEGF mRNA expression was significantly higher in the PTEN siRNA group compared with the TIMP-1-1S/HKCs and control siRNA group. However, the VEGF mRNA expression demonstrated no significant difference in the latter two groups $(\mathrm{P}>0.05)$. There was no significant difference in the expression of TIMP-1 mRNA among the three groups (Figure 8).

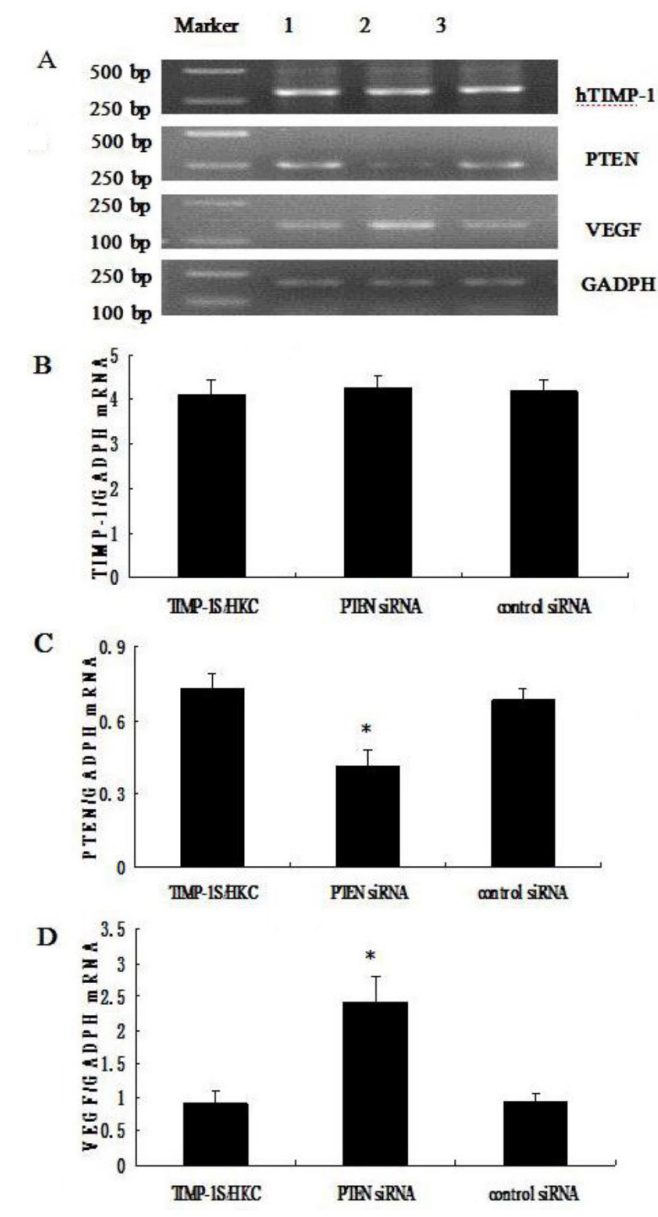

Figure 8. Expression of TIMP-1, PTEN, and VEGF mRNA. A. Lane 1: TIMP-1S/HKCs, lane 2: PTEN siRNA, and lane 3: control siRNA. B.-D. Histograms of image analysis results. ${ }^{*} \mathrm{P}<0.05$, compared with the TIMP-1S/HKCs and control siRNA group. HKCs, human kidney cells. 


\section{DISCUSSION}

Impaired microangiogenesis occurs in the development of chronic progressive nephropathy (Otrock, et al., 2007; Gao, et al., 2011) and kidney aging (Kang et al., 2001; Zhang et al., 2003; Long et al., 2004). In an age-dependent nephropathy model, decreased expression of VEGF was proven to be related to impaired angiogenesis. Previous research has revealed that the expression level of TIMP-1 was considerably elevated in the aging process in human TIMP-1 transgenic mice as compared with wild-type mice. In addition, the glomerular and peritubular capillary density decreased significantly. At the same time, PTEN expression was greatly upregulated and VEGF expression was down-regulated in the TIMP-1 transgenic mice. The results suggested that highly expressed TIMP-1 might contribute to aging-related impairment of angiogenesis via up-regulating PTEN expression during renal senescence (Zhang et al., 2003).

VEGF is an important pro-angiogenic factor. Vascular endothelial growth factor receptors (VEGFRs) include Flt-1, Flk-1, and Flt-4. Flt-1 and Flk-1 can be produced by most vascular endothelial cells except that Flt-4 is only expressed on lymphatic endothelial cells. Studies have indicated that Flk-1 is the main mediator to promote endothelial cell division, proliferation, and survival, as well as microvascular permeability. It has been proven that Flt-1 has a dual function in angiogenesis, acting in a positive or negative manner in different circumstances. Especially during early stages of embryonic development, Flt-1 exerts its negative regulation on angiogenesis by preventing VEGF from binding to Flk-1. Recent studies have shown that the expression of VEGF can be induced by hypoxia and its transcription can be directly activated by hypoxia-inducible transcription factor-1 (HIF-1). Nevertheless, up-regulation of VEGF is not only mediated by HIF1, but also by the SP-1 and ras oncogenes (Lee et al., 2012; Zhou and Tu, 2012). Furthermore, transcription of VEGF was found to be blocked by the tumor suppressor genes p53 and p57. PTEN is a new tumor suppressor gene that encodes a dual-specificity phosphatase with both protein and lipid phosphatase activities. PTEN lipid phosphatase dephosphorylates membrane-bound PIP3, blocking Akt kinase activity and its downstream kinase activity, thereby exerting its negative regulation on the cell cycle and proliferation. PTEN plays an important role in endothelial and epithelial cells. In endothelial cells, overexpressed PTEN can inhibit cell survival and angiogenesis, activating caspase-3 and thereby inducing apoptosis (Huang and Kontos, 2002). In epithelial cells, overexpressed PTEN suppresses cell proliferation and induces apoptosis via up-regulating apoptosis-related proteins (Wu et al., 2007).

In this study, after the HKCs were transfected with sense and antisense TIMP-1 and subjected to enzyme inhibitors, significantly higher PTEN expression was observed in the sense TIMP-1 transfected group than in the normal non-transfected and empty vector transfected groups $(\mathrm{P}$ $<0.05)$. However, PTEN expression decreased in the antisense TIMP-1 transfected group $(P<0.05)$. In this experiment, PTEN siRNA was transfected into HKCs stably expressing human TIMP-1S, so as to further explore the effect of sense TIMP-1 transfection on PTEN expression in HKCs. The results suggested that PTEN expression was significantly down-regulated and VEGF expression was upregulated in the PTEN siRNA transfection group compared with the normal non-transfected and control siRNA groups $(P<0.05)$. The findings indicated that TIMP-1 might exert its anti-angiogenic function by upregulating PTEN expression as well as down-regulating VEGF expression. Further studies have identified that the anti-angiogenic function of TIMP-1 is completely independent of MMP inhibitory activity, which was consistent with the report by Akahane et al. (2004).

Angiogenesis is a pathophysiological process in which endothelial cell proliferation and migration occur first, and new blood vessels form at the end stage. Furthermore, in angiogenesis, 
MMPs might promote angiogenesis by degrading the extracellular matrix, promoting migration of endothelial cells as well as releasing matrix debris. Both MMP-2 and MMP-9 can degrade capillary basement membranes and promote the migration of endothelial cells, thereby being involved in angiogenesis (Nguyen et al., 2001). In a multi-stage carcinogenesis mouse model study, Bergers et al. (2000) confirmed that MMP-9 initiated angiogenesis promoted VEGF bioavailability and VEGF receptor activation in endothelial cells. Additionally, Hashimoto et al. (2002) claimed that MMPs might activate VGEF through degrading connective tissue growth factor. TIMP-1 is an inhibitor of MMPs. Therefore, we speculated that the anti-angiogenic function of TIMP-1 could also be induced by MMP inhibitory activity.

In conclusion, this study showed that highly expressed TIMP-1 could inhibit angiogenesis by upregulating PTEN expression through an MMP-independent pathway and by down-regulating VEGF expression. Furthermore, as an endogenous inhibitor of MMPs, TIMP-1 might modulate angiogenesis via MMP inhibitory activity. We also observed that TIMP-1 was possibly involved in the MMP pathway to regulate angiogenesis in this study. Therefore, the role of the anti-angiogenic function of TIMP-1 in the progression of kidney disease still needs to be further explored and elucidated.

\section{Conflicts of interest}

The authors declare no conflict of interest.

\section{REFERENCES}

Akahane T, Akahane M, Shah A, Connor CM, et al. (2004). TIMP-1 inhibits microvascular endothelial cell migration by MMPdependent and MMP-independent mechanisms. Exp. Cell Res. 301: 158-167.

Bergers G, Brekken R, McMahon G, Vu TH, et al. (2000). Matrix metalloproteinase-9 triggers the angiogenic switch during carcinogenesis. Nat. Cell Biol. 2: 737-744.

Gao G, Zheng LL, Yang J, Li ZZ, et al. (2011). Effects of fluvastatin on the expression of matrix metalloproteinase 1, tissue inhibitors of matrix metalloproteinase 1 and intracellular adhesion molecule 1 induced by visfatin in cultured human umbilical vein endothelial cells. Chin. J. Arterioscler. 19: 121-124.

Hashimoto G, Inoki I, Fujii Y, Aoki T, et al. (2002). Matrix metalloproteinases cleave connective tissue growth factor and reactivate angiogenic activity of vascular endothelial growth factor 165. J. Biol. Chem. 277: 36288-36295.

Huang $\mathrm{J}$ and Kontos CD (2002). PTEN modulates vascular endothelial growth factor-mediated signaling and angiogenic effects. J. Biol. Chem. 277: 10760-10766.

Huang JQ, Jiao YT, Li L, et al. (2012). siRNA enterference of CTGF and TIMP-1 on rat hepatic stellate cell collagen secretions. J. Huazhong Univ. Sci. Technol. (Health Sciences) 41: 22-24. (Ref. não encontrada)

Kang DH, Anderson S, Kim YG, Mazzalli M, et al. (2001). Impaired angiogenesis in the aging kidney: vascular endothelial growth factor and thrombospondin-1 in renal disease. Am. J. Kidney Dis. 37: 601-611.

Kang DH, Kanellis J, Hugo C, Truong L, et al. (2002). Role of the microvascular endothelium in progressive renal disease. J. Am. Soc. Nephrol. 13: 806-816.

Lee MH, Choi EN, Jeon YJ and Jung SC (2012). Possible role of transforming growth factor- $\beta 1$ and vascular endothelial growth factor in Fabry disease nephropathy. Int. J. Mol. Med. 30: 1275-1280.

Long DA, Price KL and Mu W (2004). Angiopoietin-1, a vascular growth factor with a potentially novel role in aging (abstract). J. Am. Soc. Nephrol. 15: 477A.

Nguyen M, Arkell J and Jackson CJ (2001). Human endothelial gelatinases and angiogenesis. Int. J. Biochem. Cell Biol. 33: 960-970.

Otrock ZK, Makarem JA and Shamseddine Al (2007). Vascular endothelial growth factor family of ligands and receptors: Review. Blood Cells Mol. Dis. 38: 258-268.

Wu ZX, Song TB, Li DM, Zhang XT, et al. (2007). Overexpression of PTEN suppresses growth and induces apoptosis by inhibiting the expression of survivin in bladder cancer cells. Tumour Biol. 28: 9-15.

Zhang XG, Chen XM, Zhang L, Lv Y, et al. (2003). Research of the expression of vascular endothelial growth factor and its receptors in renal tissue of rats with aging. Chin. J. Geriatrics 22: 25-29.

Zhou Y and Tu GJ (2012). Construction of TIMP-1 gene recombinant adenovirus vector. J. China Med. Univ. 41: 805-808, 812. 